\title{
235.
}

\section{SUR QUELQUES FORMULES POUR LA TRANSFORMATION DES INTÉGRALES ELLIPTIQUES.}

[From the Journal für die reine und angewandte Mathematik, (Crelle), tom. LV. (1858), pp. 15-24.]

EN posant dans les formules "Fund. nova p. 15, Tab. III.," $\cos \phi=x$, on obtient

$$
\frac{d y}{\sqrt{(y-\alpha)(y-\beta)(y-\gamma)(y-\delta)}}=\frac{2}{\sqrt{(\alpha-\gamma)(\beta-\delta)}+\sqrt{(\alpha-\beta)(\gamma-\delta)}} \frac{d x}{\sqrt{\left(1-x^{2}\right)\left(1-k^{2} x^{2}\right)}}
$$
où

$$
\begin{aligned}
k^{2} & =\left(\frac{\sqrt{(\alpha-\gamma)(\beta-\delta)}-\sqrt{(\alpha-\beta)(\gamma-\delta)}}{\sqrt{(\alpha-\gamma)(\beta-\delta)+\sqrt{(\alpha-\beta)(\gamma-\delta)}})^{2}}\right. \\
\frac{1-x}{1+x} & =\frac{(\alpha-\beta)(\beta-\delta)}{(\alpha-\gamma)(\gamma-\delta)} \frac{(y-\gamma)}{(\beta-y)}
\end{aligned}
$$

Maintenant soit

$$
(y-\alpha)(y-\beta)(y-\gamma)(y-\delta)=(a, b, c, d, e \gamma y, 1)^{4}
$$

et proposons-nous d'introduire dans les formules les coefficients $(a, b, c, d, e)$ au lieu des racines $\alpha, \beta, \gamma, \delta$. En renvoyant d'ailleurs à la Note sur les covariants d'une fonction quadratique, cubique ou biquadratique insérée dans ce Journal, t. L. (1855), p. 285, [135], je pose pour abréger

$$
\begin{aligned}
& (\alpha-\beta)(\gamma-\delta)=B \\
& (\alpha-\gamma)(\delta-\beta)=C \\
& (\alpha-\delta)(\beta-\gamma)=D
\end{aligned}
$$


de sorte que l'on a identiquement

$$
B+C+D=0 .
$$

Les invariants $I, J$ sont des fonctions rationnelles des quantités $B, C, D$; en effet on a

$$
\begin{aligned}
B^{2}+C^{2}+D^{2} & =24 I, \\
(B-C)(C-D)(D-B) & =432 J, \\
B^{2} C^{2} D^{2} & =256\left(I^{2}-27 J^{3}\right) .
\end{aligned}
$$

Cela étant, nous avons

$$
k^{2}=\left(\frac{i \sqrt{ } C-\sqrt{ } B}{i \sqrt{ } C+\sqrt{ } B}\right)^{2}
$$

ce qui donne d'abord

$$
k^{4}+14 k^{2}+1=16 \frac{B^{2}+B C+C^{2}}{(i \sqrt{ } C+\sqrt{ } B)^{4}},
$$

ou, à cause de $B+C+D=0$, d'où $B^{2}+C^{2}+D^{2}=2\left(B^{2}+B C+C^{2}\right)$,

$$
k^{4}+14 k^{2}+1=\frac{8\left(B^{2}+C^{2}+D^{2}\right)}{(i \sqrt{ } C+\sqrt{ } B)^{4}} .
$$

On trouve ensuite

$$
1-k^{2}=\frac{4 i \sqrt{B C}}{(i \sqrt{ } C+\sqrt{ } B)^{4}}
$$

et de là

$$
k^{2}\left(1-k^{2}\right)^{4}=\frac{(i \sqrt{ } C-\sqrt{ } B)^{2}}{(i \sqrt{ } C+\sqrt{ } B)^{2}} \cdot \frac{256 B^{2} C^{2}}{(i \sqrt{ } C+\sqrt{ } B)^{8}},
$$

ou en multipliant le numérateur et le dénominateur par $(i \sqrt{ } C+\sqrt{ } B)^{2}$, et posant $D^{2}$ au lieu de $(B+C)^{2}$, on obtient

$$
k^{2}\left(1-k^{2}\right)^{4}=\frac{256 B^{2} C^{2} D^{2}}{(i \sqrt{ } C+\sqrt{ } B)^{12}} .
$$

Ces équations donnent

$$
\frac{k^{2}\left(1-k^{2}\right)^{4}}{\left(k^{4}+14 k^{2}+1\right)^{3}}=\frac{B^{2} C^{2} D^{2}}{2\left(B^{2}+C^{2}+D^{2}\right)^{3}}
$$

ou, en posant

On trouve aussi

$$
\begin{aligned}
N & =\frac{27}{4} \frac{1}{1-\frac{27 J^{2}}{I^{3}}} \\
\frac{k^{2}\left(1-k^{2}\right)^{4}}{\left(k^{4}+14 k^{2}+1\right)^{3}} & =\frac{1}{16 N} .
\end{aligned}
$$

$$
k^{4}+14 k^{2}+1=\frac{192 I}{(i \sqrt{ } C+\sqrt{ } B)^{4}},
$$


ce qui donne

$$
\frac{2}{i \sqrt{ } C+\sqrt{ } B}=\sqrt[4]{\frac{k^{4}+14 k^{2}+1}{12 I}}
$$

et la formule de transformation devient ainsi

$$
\frac{d y}{\sqrt{\left(a, b, c, d, e^{\gamma} y, 1\right)^{4}}}=\sqrt[4]{\frac{k^{4}+14 k^{2}+1}{12 I}} \frac{d x}{\sqrt{\left(1-x^{2}\right)\left(1-k^{2} x^{2}\right)}},
$$

le module $k$ étant déterminé par l'équation

$$
\frac{k^{2}\left(1-k^{2}\right)^{4}}{\left(k^{4}+14 k^{2}+1\right)^{3}}=\frac{1}{16 N}
$$

où

$$
N=\frac{27}{4} \frac{1}{1-\frac{27 J^{2}}{I^{3}}}
$$

Cela revient à une formule que j’ai donnée dans le mémoire intitulé, "On the Reduction of $d u \div \sqrt{ } U$ when $U$ is a Function of the Fourth Order," Camb. and Dublin Math. Journal, t. I. (1846), p. 70, [33], et de laquelle j’ai déduit des conséquences que je vais reproduire ici. En effet l'équation en $k$ peut s'écrire sous la forme

$$
\left(k^{4}+14 k^{2}+1\right)^{3}-16 N k^{2}\left(k^{2}-1\right)^{4}=0,
$$

c'est-à-dire

$$
\left(k^{2}+\frac{1}{k^{2}}+14\right)^{3}-16 N\left(k-\frac{1}{k}\right)^{4}=0
$$

en écrivant

$$
k-\frac{1}{k}=\frac{4}{\sqrt{9-1}}
$$

on obtient pour 9 l'équation très-simple

$$
9^{3}-N(9-1)=0
$$

et on a ensuite

$$
k^{2}=\frac{7+9+4 \sqrt{3}+9}{9-1}
$$

ce qui donne aussi

$$
k=\frac{2+\sqrt{3+9}}{\sqrt{9-1}}
$$

Soit $k=\beta^{2}$ l'une des valeurs de $k$, l'équation en $k$ devient

$$
\frac{\left(k^{4}+14 k^{2}+1\right)^{3}}{k^{2}\left(k^{2}-1\right)^{4}}=\frac{\left(\beta^{8}+14 \beta^{4}+1\right)^{3}}{\beta^{4}\left(\beta^{4}-1\right)^{4}},
$$


équation à laquelle on satisfait, en outre, par la valeur $k=\left(\frac{1-\beta}{1+\beta}\right)^{2}$. En effet cette valeur donne

$$
k^{4}+14 k^{2}+1=\frac{16\left(\beta^{8}+14 \beta^{4}+1\right)^{3}}{(1+\beta)^{8}}, \quad k^{2}-1=-\frac{8 \beta\left(1+\beta^{2}\right)}{(1+\beta)^{4}},
$$

expressions qui rendent l'équation identique. Cela fait voir que l'équation peut s'écrire sous la forme

$$
\begin{aligned}
& \left(k^{4}+14 k^{2}+1\right)^{4}-k^{2}\left(k^{2}-1\right)^{4} \frac{\left(\beta^{8}+14 \beta^{4}+1\right)^{3}}{\beta^{4}\left(\beta^{4}-1\right)^{4}} \\
& \quad=\left(k^{2}-\beta^{4}\right)\left(k^{2}-\frac{1}{\beta^{4}}\right)\left(k^{2}-\left(\frac{1-\beta}{1+\beta}\right)^{4}\right)\left(k^{2}-\left(\frac{1+\beta}{1-\beta}\right)^{4}\right)\left(k^{2}-\left(\frac{1-\beta i}{1+\beta i}\right)^{4}\right)\left(k^{2}-\left(\frac{1+\beta i}{1-\beta i}\right)^{4}\right)
\end{aligned}
$$

et les racines de l'équation en $k^{2}$ sont

$$
\beta^{4}, \quad \frac{1}{\beta^{4}}, \quad\left(\frac{1-\beta}{1+\beta}\right)^{4}, \quad\left(\frac{1+\beta}{1-\beta}\right)^{4}, \quad\left(\frac{1-\beta i}{1+\beta i}\right)^{4}, \quad\left(\frac{1+\beta i}{1-\beta i}\right)^{4},
$$

ce'qui s'accorde avec un résultat obtenu par Abel (voir les œuvres d'Abel t. I. p. 310 [Ed. 2, p. 4.59]).

Je fais observer à présent qu'en écrivant

$$
9=\frac{3 \varpi}{2 \sigma-3}
$$

on obtient pour $\varpi$ l'équation $(27-4 N) \varpi^{3}+27 N(\varpi-1)=0$, qui, en posant $M=\frac{-27 N}{27-4 N}$, ou ce qui est la même chose

$$
M=\frac{I^{3}}{4 J^{2}}
$$

devient

$$
\varpi^{3}-M(\varpi-1)=0 .
$$

Cette équation est précisément la même que celle à laquelle je suis parvenu dans la Note sur les covariants etc. ci-dessus citée. La quantité ø est liée au module $k$ par la relation

$$
k=\frac{3 \sqrt{\varpi-1}+2 \sqrt{2 \sigma-3}}{\sqrt{\varpi+3}} .
$$

On peut introduire $M$ au lieu de $N$ dans l'équation en $k$, et en combinant les formules précédemment obtenues, on trouve

où

$$
\frac{d y}{\sqrt{\left(a, b, c, d, e \gamma(y, 1)^{4}\right.}}=\sqrt[4]{\frac{k^{4}+14 k^{2}+1}{12 I}} \frac{d x}{\sqrt{\left(1-x^{2}\right)\left(1-k^{2} x^{2}\right)}}
$$

$$
\frac{27\left(1+14 k^{2}+k^{4}\right)^{3}}{\left(1+k^{2}\right)^{2}\left(1-34 k^{2}+k^{4}\right)^{4}}=4 M, \quad M=\frac{I^{3}}{4 J^{2}},
$$


ou ce qui revient à la même chose,

$$
k=\frac{3 \sqrt{\varpi-1}+2 \sqrt{2 \varpi-3}}{\sqrt{\varpi+3}}, \quad \varpi^{3}-M(\varpi-1)=0 .
$$

De l'équation entre $k$ et 9 on déduit facilement la relation

$$
\frac{k^{4}+14 k^{2}+1}{k^{4}-34 k^{2}+1}=\frac{-9}{29-3}
$$

et en substituant dans cette équation pour 9 sa valeur on obtient

$$
\frac{1-34 k^{2}+k^{4}}{-3\left(1+14 k^{2}+k^{4}\right)}=\frac{1}{\varpi}
$$

ce qui est encore une forme de la relation entre $k$ et $\varpi$.

\section{II}

On peut obtenir les résultats qui viennent d'être déduits de la formule de Jacobi, en prenant pour point de départ la transformation d'une fonction du quatrième ordre dans sa forme canonique. Je suppose d'abord que l'on a identiquement

$$
\begin{aligned}
\left(a, b, c, d, e \gamma(x, y)^{4}\right. & =(\lambda x+\mu y)^{4}+\left(\lambda^{\prime} x+\mu^{\prime} y\right)^{4}+6 \theta(\lambda x+\mu y)^{2}\left(\lambda^{\prime} x+\mu^{\prime} y\right)^{2} \\
& =x_{1}^{4}+y_{1}{ }^{4}+6 \theta x_{1}^{2} y_{1}^{2}
\end{aligned}
$$

Cela étant, je pose $\lambda \mu^{\prime}-\lambda^{\prime} \mu=\Lambda$, et je forme les covariants des deux expressions; on obtient par la propriété fondamentale de ces fonctions

$$
\begin{aligned}
& I=\Lambda^{4}\left(1+3 \theta^{2}\right) \\
& J=\Lambda^{6}\left(\theta-\theta^{3}\right) \\
& U=x_{1}^{4}+y_{1}^{4}+6 \theta x_{1}^{2} y_{1}^{2} \\
& H=\Lambda^{2}\left\{\theta x_{1}^{4}+\theta y_{1}^{4}+\left(1-3 \theta^{2}\right) x_{1}^{2} y_{1}^{2}\right\} \\
& \Phi=\Lambda^{3}\left(9 \theta^{2}-1\right) x_{1} y_{1}\left(x_{1}^{4}-y_{1}^{4}\right) .
\end{aligned}
$$

De ces relations on tire

$$
\begin{aligned}
& \frac{J}{I}=\Lambda^{2} \frac{\theta-\theta^{3}}{1+3 \theta^{2}}, \\
& \frac{J^{2}}{I^{3}}=\frac{\left(\theta-\theta^{3}\right)^{2}}{\left(1+3 \theta^{2}\right)^{3}}
\end{aligned}
$$

de sorte qu'en posant

$$
M=\frac{I^{3}}{4 J^{2}},
$$


on aura pour déterminer $\theta$, l'équation

$$
\frac{\left(1+3 \theta^{2}\right)^{3}}{\left(\theta-\theta^{3}\right)^{2}}=4 M,
$$

et pour déterminer $x_{1}, y_{1}$ les équations

$$
\begin{aligned}
U & =x_{1}^{4}+y_{1}^{4}+\quad 6 \theta x_{1}^{2} y_{1}^{2}, \\
\frac{I}{J} \frac{1-\theta^{2}}{1+3 \theta^{2}} H & =x_{1}^{4}+y_{1}{ }^{4}+\frac{1-3 \theta^{2}}{\theta} x_{1}^{2} y_{1}^{2} .
\end{aligned}
$$

Je fais observer qu'en désignant par $\lambda$ un coefficient tel que $U+6 \lambda H$ soit un carré parfait, on obtient pour $\lambda$ l'équation

$$
1-9 \lambda^{2} I-54 \lambda^{3} J=0 .
$$

En effet le cubicovariant $\Phi$ d'une fonction qui est un carré parfait est identiquement égal à zéro, et le cubicovariant de la fonction $U+6 \lambda H$ est $\left(1-9 \lambda^{2} I-54 \lambda^{3} J\right) \Phi$, on a donc pour $\lambda$ l'équation qui vient d'être proposée; cela posé, en observant que

$$
U-\frac{I}{J} \frac{1-\theta^{2}}{1+3 \theta^{2}} H=\frac{9 \theta^{2}-1}{\theta} x_{1}^{2} y_{1}^{2},
$$

on aura pour une des valeurs de $\lambda$,

et en effet cette valeur donne

$$
\lambda=-\frac{I}{6 J} \frac{1-\theta^{2}}{1+3 \theta^{2}}
$$

$$
1-\frac{I^{3}}{J^{2}} \frac{\left(\theta-\theta^{3}\right)^{2}}{\left(1+3 \theta^{2}\right)^{3}}=0
$$

ce qui est l'équation en $\theta$. Cela étant, je pose

$$
\lambda=-\frac{I}{6 J} \frac{1}{\varpi_{3}}
$$

alors $\varpi_{3}$ sera une racine de l'équation

on obtient

$$
\varpi_{3}-M(\varpi-1)=0
$$

$$
\varpi_{3}=\frac{1+3 \theta^{2}}{1-\theta^{2}}
$$

et de là

$$
\theta^{2}=\frac{\varpi_{3}-1}{\varpi_{3}+3}
$$

Soient $\varpi_{1}, \varpi_{2}$ les deux autres racines de l'équation en $\varpi$, on aura

$$
\begin{aligned}
\varpi_{1}+\varpi_{2} & =-\varpi_{3}, \\
\varpi \varpi_{2} & =-\frac{\varpi_{3}{ }^{2}}{\varpi_{3}-1},
\end{aligned}
$$

C. IV. 
ce qui donne

On pourra donc écrire

$$
\left(\varpi_{1}-\varpi_{2}\right)^{2}=\frac{\varpi_{3}^{2}\left(\varpi_{3}+3\right)}{\varpi_{3}-1}=\frac{\varpi_{3}^{2}}{\theta^{2}}
$$

$$
\theta=\frac{-\varpi_{3}}{\varpi_{2}-\varpi_{1}}
$$

et au moyen des valeurs de $U, H$ on obtient, par une très-simple réduction, les trois équations suivantes

$$
\begin{array}{lr}
I H-\varpi_{1} J U= & \left(\varpi_{3}-\varpi_{1}\right) J\left(x_{1}{ }^{2}+y_{1}{ }^{2}\right)^{2}, \\
I H-\varpi_{2} J U=- & \left(\varpi_{2}-\varpi_{3}\right) J\left(x_{1}{ }^{2}-y_{1}{ }^{2}\right)^{2}, \\
I H-\varpi_{3} J U=-\frac{\left(\varpi_{2}-\varpi_{3}\right)\left(\varpi_{3}-\varpi_{1}\right)}{\sigma_{1}-\varpi_{2}} J .4 x_{1}^{2} y_{1}{ }^{2},
\end{array}
$$

dont deux quelconques donnent les valeurs de $x_{1}, y_{1}$; ainsi on a obtenu la solution complète du problème de la réduction de la fonction $\left(a, b, c, d, e \gamma(x, y)^{4}\right.$ à la forme canonique.

Je fais observer que ces équations montrent a posteriori que les expressions $I H-\varpi_{1} J U, I H-\varpi_{2} J U, I H-\varpi_{3} J U$ sont toutes les trois des carrés de fonctions quadratiques. Je fais observer, en outre, que si l'on forme la valeur de l'expression

$$
\left(\varpi_{2}-\varpi_{3}\right) \sqrt{I H-\varpi_{1} J U}+\left(\varpi_{3}-\varpi_{1}\right) \sqrt{I H-\varpi_{2} J U}+\left(\varpi_{1}-\varpi_{2}\right) \sqrt{I H-\varpi_{3} J U},
$$

en posant pour un moment $\varpi_{2}-\varpi_{3}=\alpha, \varpi_{3}-\varpi_{1}=\beta, \varpi_{1}-\varpi_{2}=\gamma$, l'expression dont il s'agit sera égale, à un facteur constant près, à

$$
\sqrt{ } \alpha\left(x_{1}^{2}+y_{1}^{2}\right)+i \sqrt{ } \beta\left(x_{1}^{2}-y_{1}^{2}\right)+i \sqrt{ } \gamma \cdot 2 x_{1} y_{1}
$$

ou, ce qui est la même chose, à

$$
\left(\sqrt{ } \alpha+i \sqrt{ } \beta, i \sqrt{ } \gamma, \sqrt{ } \alpha-i \sqrt{ } \beta \gamma\left(x_{1}, y_{1}\right)^{2} .\right.
$$

Or, cette fonction doit être un carré parfait, ce qu'on reconnaît en effet, en se rappelant que

$$
(\sqrt{ } \alpha+i \sqrt{ } \beta)(\sqrt{ } \alpha-i \sqrt{ } \beta)-(i \sqrt{ } \gamma)^{2}=\alpha+\beta+\gamma=0 .
$$

$\mathrm{Sa}$ racine carrée sera, à un facteur constant près, $(\sqrt{ } \alpha+i \sqrt{ } \beta) x_{1}+i \sqrt{ } \gamma y_{1}$, et cette dernière fonction sera un des facteurs linéaires de $x_{1}^{4}+y_{1}^{4}+6 \theta x_{1}^{2} y_{1}^{2}$. Pour vérifier cela je pose $x_{1}^{4}+y_{1}^{4}+6 \theta x_{1}^{2} y_{1}^{2}=0$, ce qui donne

$$
x_{1}^{2}+\left(3 \theta+\sqrt{9 \theta^{2}-1}\right) y_{1}^{2}=0,
$$

et $\quad x_{1}-\left(\sqrt{\frac{3 \theta+1}{2}}+\sqrt{\frac{3 \theta-1}{2}}\right) y_{1}=0 . \quad$ Or $3 \theta+1=\frac{-3 \varpi_{3}}{\varpi_{2}-\varpi_{1}}+1=\frac{-3 \varpi_{3}+\varpi_{2}-\varpi_{1}}{\varpi_{2}-\varpi_{1}}$

$=\frac{-2 \varpi_{3}+2 \varpi_{2}-\left(\varpi_{1}+\varpi_{2}+\varpi_{3}\right)}{\varpi_{2}-\varpi_{1}}=\frac{2 \varpi_{2}-2 \varpi_{3}}{\varpi_{2}-\varpi_{3}}=\frac{2 \alpha}{-\gamma}$, c'est-à-dire $\sqrt{\frac{3 \theta+1}{2}}=\frac{\sqrt{ } \alpha}{i \sqrt{ } \gamma}$, et de même $\sqrt{\frac{3 \theta-1}{2}}=\frac{i \sqrt{ } \beta}{i \sqrt{ } \gamma}$, le facteur linéaire sera done $i \sqrt{ } \gamma \cdot x_{1}-(\sqrt{ } \alpha+i \sqrt{ } \beta) y_{1}$ ou, ce qui revient à la même chose, $(\sqrt{ } \alpha+i \sqrt{ } \beta) x_{1}+i \sqrt{ } \gamma y_{1}$, ce qu'il s'agissait de démontrer. 
III.

Pour obtenir la formule qui sert à la transformation d'une intégrale elliptique, il faut présenter les résultats précédemment obtenus sous une forme un peu différente. J'écris

où

$$
\begin{aligned}
(a, b, c, d, e \gamma y, 1)^{4} & =(\lambda+\mu y)^{4}-\left(1+k^{2}\right)(\lambda+\mu y)^{2}\left(\lambda^{\prime}+\mu^{\prime} y\right)^{2}+k^{2}\left(\lambda^{\prime}+\mu^{\prime} y\right)^{4} \\
& =(\lambda+\mu y)^{4} \cdot\left(1-x^{2}\right)\left(1-k^{2} x^{2}\right),
\end{aligned}
$$

$$
x=\frac{\lambda^{\prime}+\mu^{\prime} y}{\lambda+\mu y} .
$$

Cela posé, en remplaçant comme auparavant $\lambda \mu^{\prime}-\lambda^{\prime} \mu$ par $\Lambda$, et en formant les covariants, on obtient

$$
\begin{aligned}
& I=\frac{1}{12} \Lambda^{4}\left(1+14 k^{2}+k^{4}\right) \\
& J=\frac{1}{216} \Lambda^{6}\left(1+k^{2}\right)\left(1-34 k^{2}+k^{4}\right) \\
& U=(\lambda+\mu y)^{4}\left\{1-\left(1+k^{2}\right) x^{2}+k^{2} x^{4}\right\} \\
& H=-\frac{1}{12} \Lambda^{2}(\lambda+\mu y)^{4}\left\{2\left(1+k^{2}\right)-\left(1-10 k^{2}+k^{4}\right) x^{2}+2 k^{2}\left(1+k^{2}\right) x^{4}\right\} \\
& \Phi=\frac{1}{4} \Lambda^{3}(\lambda+\mu y)^{6}\left(1-k^{2}\right)^{3} x\left(1-k^{2} x^{4}\right) .
\end{aligned}
$$

On a d'abord

$$
\frac{27\left(1+14 k^{2}+k^{4}\right)^{3}}{\left(1+k^{2}\right)^{2}\left(1-34 k^{2}+k^{4}\right)^{2}}=4 M
$$

en posant comme auparavant $M=\frac{I^{3}}{4 J^{2}}$; on obtient ensuite

$$
\Lambda^{4}=\frac{12 I}{1+14 k^{2}+k^{4}}
$$

et en remarquant que l'équation entre $x$ et $y$ donne

on trouve

$$
d x=\frac{\left(\lambda \mu^{\prime}-\lambda^{\prime} \mu\right) d y}{(\lambda+\mu y)^{2}}=\frac{\Lambda d y}{(\lambda+\mu y)^{2}}
$$

$$
\frac{d y}{\sqrt{(a, b, c, d, e \gamma y, 1)^{4}}}=\frac{1}{\Lambda} \frac{d x}{\sqrt{\left(1-x^{2}\right)\left(1-k^{2} x^{2}\right)}},
$$

c'est-à-dire

$$
\frac{d y}{\sqrt{(a, b, c, d, e \gamma y, 1)^{4}}}=\sqrt[4]{\frac{k^{4}+14 k^{2}+1}{12 I}} \frac{d x}{\sqrt{\left(1-x^{2}\right)\left(1-k^{2} x^{2}\right)}},
$$

ce qui s'accorde avec la formule ci-dessus trouvée; pour compléter la solution, je pose

$$
\frac{1-34 k^{2}+k^{4}}{-3\left(1+14 k^{2}+k^{4}\right)}=\frac{1}{\sigma_{3}}
$$


$\varpi_{3}$ sera une des racines de l'équation

$$
\varpi_{3}-M(\varpi-1)=0
$$

en représentant par $\varpi_{1}, \varpi_{2}$ les deux autres racines, on obtient

$$
\begin{aligned}
& 2 \varpi_{1}=-\varpi_{3} \frac{1+6 k+k^{2}}{1+k^{2}}, \\
& 2 \varpi_{2}=-\varpi_{3} \frac{1-6 k+k^{2}}{1+k^{2}},
\end{aligned}
$$

et ensuite

$$
\begin{aligned}
& I H-\varpi_{1} J U=J(\lambda+\mu y)^{4}\left(\varpi_{3}-\varpi_{1}\right)\left(1+k x^{2}\right)^{2}, \\
& I H-\varpi_{2} J U=J(\lambda+\mu y)^{4}\left(\varpi_{3}-\varpi_{2}\right)\left(1-k x^{2}\right)^{2}, \\
& I H-\varpi_{3} J U=J \frac{\left(\varpi_{3}-\varpi_{1}\right)\left(\varpi_{3}-\varpi_{2}\right)}{\varpi_{1}-\varpi_{2}}(\lambda+\mu y)^{4} .4 k x^{2} .
\end{aligned}
$$

Donc en posant $\varpi_{2}-\varpi_{3}=\alpha, \varpi_{3}-\varpi_{1}=\beta, \varpi_{1}-\varpi_{2}=\gamma$, on a

$$
\begin{aligned}
\left(\varpi_{2}-\varpi_{3}\right) \sqrt{I H-\varpi_{1} J U}+\left(\varpi_{3}-\varpi_{1}\right) \sqrt{I H-\varpi_{2} J U}+\left(\varpi_{1}-\varpi_{2}\right) \sqrt{\left.I H-\varpi_{3} J U\right)} \\
=J(\lambda+\mu y)^{4} \sqrt{\alpha \beta}(\sqrt{ } \alpha+i \sqrt{ } \beta+i \sqrt{ } \gamma x \sqrt{ } k)^{2},
\end{aligned}
$$

de même

$$
\begin{array}{r}
\left(\varpi_{2}-\varpi_{3}\right) \sqrt{I H-\varpi_{1} J U}+\left(\varpi_{3}-\varpi_{1}\right) \sqrt{I H-\varpi_{2} J U}-\left(\varpi_{1}-\varpi_{2}\right) \sqrt{I H-\varpi_{3} J U} \\
=J(\lambda+\mu y)^{4} \sqrt{\alpha \beta}(\sqrt{ } \alpha+i \sqrt{ } \beta-i \sqrt{ } \gamma x \sqrt{ } k)^{2},
\end{array}
$$

et de là enfin, en remplaçant $\alpha, \beta, \gamma$ par leurs valeurs,

$$
\begin{array}{r}
\frac{\left(\varpi_{2}-\varpi_{3}\right) \sqrt{I H-\varpi_{1} J U}+\left(\varpi_{3}-\varpi_{1}\right) \sqrt{I H-\varpi_{2} J U}+\left(\varpi_{1}-\varpi_{2}\right) \sqrt{I H-\varpi_{3} J U}}{\left(\varpi_{2}-\varpi_{3}\right) \sqrt{I H-\varpi_{1} J U}+\left(\varpi_{3}-\varpi_{1}\right) \sqrt{I H-\varpi_{2} J U-\left(\varpi_{1}-\varpi_{2}\right) \sqrt{I H-\varpi_{3} J U}}} \\
=\left(\frac{\sqrt{\varpi_{2}-\varpi_{3}}+i \sqrt{\varpi_{3}-\varpi_{1}}+i \sqrt{\varpi_{1}-\varpi_{2}} x \sqrt{k}}{\sqrt{\varpi_{2}-\varpi_{3}}+i \sqrt{\varpi_{3}-\varpi_{1}}-i \sqrt{\varpi_{1}-\varpi_{2}} x \sqrt{k}}\right)^{2},
\end{array}
$$

équation dont le premier membre est le carré d'une fraction rationnelle de la forme

$$
\frac{A+B x}{C+\dot{D} x}
$$

IV.

Je terminerai ces recherches en démontrant le théorème de $M$. Hermite dont j'ai parlé dans la Note sur les covariants etc. ci-dessus citée. L'identité

$$
J U^{3}-I U^{2} H+4 H^{3}=-\Phi^{2}
$$


peut être mise sous la forme

$$
-J+I \cdot \frac{H}{U}-4 \frac{H^{3}}{U^{3}}=\left(\frac{\Phi}{U^{2}}\right)^{2} \cdot U
$$

Posons maintenant

$$
z=\frac{H}{\bar{U}}
$$

formule dans laquelle je suppose qu'on ait fait. $y=1$, de manière que $U, H$ soient des fonctions rationnelles et entières de la seule variable $x$, savoir

$$
\begin{aligned}
& U=\left(a, b, c, d, e \gamma(x, 1)^{4},\right. \\
& H=\left(a c-b^{2}, \quad \frac{1}{2}(a d-b c), \quad \frac{1}{6}\left(a e+2 b d-3 c^{2}\right), \quad \frac{1}{2}(b e-c d), \quad c e-d^{2} \gamma(x, 1)^{4},\right.
\end{aligned}
$$

alors on aura

$$
\sqrt{-J+z I-4 z^{3}}=\frac{\Phi}{U^{2}} \sqrt{\left(a, b, c, d, e \gamma(x, 1)^{4}\right.}
$$

et

$$
d z=\frac{U d H-H d U}{U^{2}}
$$

En vertu de la théorie de Jacobi, $U d H-H d U$ est de la forme $M \Phi d x$, où $M$ est un facteur constant; on trouve en effet très-facilement $U d H-H d U=2 \Phi d x$, d'où l'on déduit la formule

$$
\frac{d z}{\sqrt{-J+z I-4 z^{3}}}=\frac{2 d x}{\sqrt{\left(a, b, c, d, e \gamma(x, 1)^{4}\right.}}
$$

et je fais observer que l'intégrale du premier membre se ramène immédiatement à une forme qui ne contient que la seule constante $M,=\frac{I^{3}}{4 J^{2}}$.

Londres, 9 Avril, 1856. 